\title{
ARTICLE
}

\section{Organization of olfactory centres in the malaria mosquito Anopheles gambiae}

\author{
Olena Riabinina ${ }^{1} \uparrow$, Darya Task ${ }^{1}$, Elizabeth Marr ${ }^{1}$, Chun-Chieh Lin ${ }^{1}$, Robert Alford ${ }^{2}$, David A. O'Brochta ${ }^{2}$ \\ \& Christopher J. Potter ${ }^{1}$
}

Mosquitoes are vectors for multiple infectious human diseases and use a variety of sensory cues (olfactory, temperature, humidity and visual) to locate a human host. A comprehensive understanding of the circuitry underlying sensory signalling in the mosquito brain is lacking. Here we used the Q-system of binary gene expression to develop transgenic lines of Anopheles gambiae in which olfactory receptor neurons expressing the odorant receptor co-receptor (Orco) gene are labelled with GFP. These neurons project from the antennae and maxillary palps to the antennal lobe $(A L)$ and from the labella on the proboscis to the suboesophageal zone (SEZ), suggesting integration of olfactory and gustatory signals occurs in this brain region. We present detailed anatomical maps of olfactory innervations in the $\mathrm{AL}$ and the SEZ, identifying glomeruli that may respond to human body odours or carbon dioxide. Our results pave the way for anatomical and functional neurogenetic studies of sensory processing in mosquitoes.

\footnotetext{
${ }^{1}$ The Solomon H. Snyder Department of Neuroscience, Center for Sensory Biology, Johns Hopkins University School of Medicine, 855 North Wolfe Street, 434 Rangos Building, Baltimore, Maryland 21205, USA. ${ }^{2}$ University of Maryland College Park, 9600 Gudelsky Drive, Rockville, Maryland 20850, USA. $\dagger$ Present address: MRC Clinical Sciences Centre, Imperial College London, Du Cane Road, London W12 ONN, UK. Correspondence and requests for materials should be addressed to C.J.P. (email: cpotter@jhmi.edu).
} 
A nopheles gambiae is the major insect vector of Plasmodium falciparum, the parasite that leads to malaria which is responsible for $\sim 450,000$ deaths every year ${ }^{1}$. The control of these insects is therefore an important human health issue. Currently, malaria control strategies focus on eliminating stages of parasite transmission (such as Plasmodium infection of mosquitoes), or to limit the size of mosquito populations ${ }^{2}$. However, relatively few strategies focus on interfering with the human host-seeking behavior of mosquitoes. Female A. gambiae mosquitoes are strongly anthropophilic and prefer to bloodfeed on humans over other animals. They have also been shown to use olfactory cues to locate humans $s^{3,4}$ and are likely to use olfactory cues to distinguish humans from other animals ${ }^{5-7}$. Therefore, strategies that target the mosquito's olfactory system might provide effective means to control host seeking, including the development of improved trap baits and insect repellants. However, relatively little is known about the mechanisms of olfactory processing in A. gambiae.

Knowledge about insect olfactory systems primarily stems from studies of the vinegar fly Drosophila melanogaster (for example, see ref. 8). Over the last 25 years, the molecular and cellular basis of Drosophila olfaction has been well characterized (for example, see refs 9-12). Numerous studies have elucidated the identity of Drosophila olfactory receptors and the odours that activate them, the identity and structure of the sensory neurons that express these odorant receptors (ORs), how these olfactory receptor neurons innervate the brain, all leading to an in-depth understanding of how odorant information is received, integrated and processed by the Drosophila peripheral and central nervous systems. Studies of Drosophila olfaction are facilitated by a rich repertoire of genetic tools that are not currently available in mosquitoes. The development of similar neurogenetic methods in the mosquito system would greatly advance investigation of sensory processing in this insect.

In the absence of neurogenetic methods, studies in A. gambiae have focused on a detailed characterization of the peripheral olfactory system. Chemosensory tissues in malaria mosquitoes are covered in specialized sensory hairs called sensilla that typically contain two to three olfactory receptor neurons ${ }^{13-15}$. Similar to the peripheral olfactory system in Drosophila, 3 classes of chemosensory receptors are found in the A. gambiae genome: $79 \mathrm{ORs}^{12,16,17}, 46$ ionotropic receptors (IRs) ${ }^{18,19}$ and 60 gustatory receptors $(\mathrm{GRs})^{20-23}$. In situ hybridization and single sensillum electrophysiological recordings in Drosophila, mosquitoes and other insects show that neurons expressing ORs and IRs respond to a variety of volatile odorants including many found in human body odours ${ }^{11,18,24-26}$, whereas GRs respond to a number of stimuli including tastants, pheromones, warmth and the volatile gas carbon dioxide ${ }^{22,27}$.

ORs in all insects require an obligatory OR co-receptor (Orco) for dendritic trafficking of ORs and odorant-induced signal$\operatorname{ling}^{28,29}$. Thus, all functional insect OR-expressing neurons also express the Orco gene. OR-expressing neurons have been detected by fluorescence in situ hybridization studies of Orco in the antennae and maxillary palps during the fourth instar larvae developmental stage of A. gambiae ${ }^{30}$. In the adult A. gambiae, Orco- and OR-expressing neurons have been found in three olfactory organs. Prominent Orco expression was found in the trichoid sensilla on the 2nd to 13th antennal segments in females, and on the distal club segments in males ${ }^{17}$. Orco expression has also been found in the capitate peg sensilla on the second to fourth segments of the maxillary palps in females and in the labella of the proboscis ${ }^{17,22}$. The maxillary palp capitate pegs in $A$. aegypti ${ }^{31}$ and $A$. gambiae ${ }^{22}$ also contain neurons that express a $\mathrm{CO}_{2}$-sensing GR receptor complex. Orco-expressing neurons comprise $\sim 70 \%$ of all olfactory neurons in Drosophila, suggesting
Orco is likely to be expressed in the majority of olfactory neurons in A. gambiae and thus is an attractive candidate for neurogenetic targeting in this species.

Binary expression systems, such as GAL4/UAS 32,33 and $\mathrm{QF} / \mathrm{QUAS}$ (Q-system ${ }^{34,35}$ ), are useful and versatile tools for targeting distinct neuronal subpopulations in Drosophila ${ }^{36}$. The GAL4/UAS system has been used previously in A. aegypti ${ }^{37}$, A. gambiae $^{38}$ and Anopheles stephensi ${ }^{39}$ to target tissues such as the midgut that are involved in parasite infection in the mosquito. To enable robust in vivo activity, the activation domain of GAL4 was replaced with the activation domain of the Herpes simplex VP16, to achieve satisfactory expression of a $U A S-Y F P$ reporter $^{38}$ in A. gambiae. A second generation of the QF transcriptional activator, QF2, drives strong expression of reporters without compromising cellular functions or the overall health of transgenic animals ${ }^{35}$. QF2 is also likely to be a more potent in vivo transcriptional activator than GAL4 (refs 34,35).

Here we used the Q-system to target Orco-expressing olfactory neurons in A. gambiae. We characterized a sexually dimorphic expression pattern of Orco-expressing $(\mathrm{Orco}+)$ neurons in both larvae and adult mosquitoes. This transgenic labelling of olfactory neurons allowed visualization and identification of Orco + axonal targets in the brain. Surprisingly, we found that the brain of A. gambiae contains two regions innervated by Orco + olfactory neurons. Antennal and maxillary palp neurons target the glomeruli of the antennal lobe, but Orco + olfactory neurons in the proboscis labella innervate the suboesophageal zone (SEZ) in the brain, a region likely to be involved in gustatory processing. We generated the first map of A. gambiae antennal lobe (AL) glomeruli based on olfactory receptor expression and tissue of origin. The AL contains approximately equal numbers of glomeruli innervated by Orco + and Orco-negative (Orco-) neurons. We hypothesize that the majority of Orco - glomeruli in the AL probably receive innervation from antennal olfactory neurons expressing chemosensory IR genes. We also identified the Orco - AL glomeruli probably targeted by the GR-expressing (carbon dioxide-sensing) neurons from the maxillary palp. We further generated a map of the eight glomerular structures in the SEZ formed by Orco + olfactory neurons.

These studies introduce a neurogenetic approach for investigating olfactory processing in the mosquito and suggest that the SEZ of the brain may directly integrate olfactory and gustatory cues from the proboscis during bloodfeeding.

\section{Results}

Introduction of neurogenetic tools into $A$. gambiae. The introduction of the binary Q-system into A. gambiae required the generation of two different transgenic animal lines: a promoterQF2 driver line and a QUAS-geneX effector line. The QF2 transcriptional activator binds and activates the exogenous transgenic effector gene (QUAS-geneX). Only when the two components are brought together in the same animal by genetic crosses is expression of the effector gene induced in the same pattern as the transcriptional activator. To genetically target olfactory receptor neurons, we used the presumptive enhancer and promoter regions of the Orco (AGAP002560, VectorBase v.AgamP4) gene to generate an Orco-QF2 driver construct. We PCR amplified a 9,312 bp sequence immediately upstream of the Orco ATG translation start codon and cloned it $5^{\prime}$ to QF2 in a piggyBac vector containing the synthetic $3 x P 3$ eye promoter driving a DsRed fluorescent marker (see Supplementary Fig. 1 and Methods for details). The 9,312 bp putative promoter/enhancer region was the largest PCR product $5^{\prime}$ to the Orco ATG start codon we could obtain and it is possible that the 
actual genomic region needed for Orco expression is located within a smaller portion of this sequence.

To facilitate the study of neuronal anatomy, we generated a membrane-targeted green fluorescent protein (GFP) reporter (QUAS-mCD8:GFP), which has been shown to strongly label all neuronal processes in Drosophila ${ }^{40}$. This allowed the axonal targets of the olfactory receptor neurons to be easily identified-a task not possible using existing anti-Orco antibodies. To generate the QUAS- $m C D 8: G F P$ reporter construct, we cloned 15 copies of the QUAS sequence ${ }^{34}$ followed by the Drosophila hsp70 minimal promoter into a piggyBac vector marked by a $3 \times P 3-E C F P$ marker. Immediately downstream of the $h s p 70$ promoter, we cloned a gene containing the mouse CD8 single-pass membrane protein fused to GFP (see Supplementary Fig. 1 and Methods for details). Increasing the number of transcription factor-binding sites in reporter constructs increases expression levels ${ }^{41}$ and we reasoned that using 15x QUAS instead of the standard 5x QUAS ${ }^{34}$ would lead to increased expression levels of GFP. This approach may prove particularly useful in reporting the activity of weak promoter lines, including olfactory receptor promoters ${ }^{9,10,29}$.

We generated three Orco-QF2 transgenic lines and two QUAS$m C D 8$ :GFP transgenic lines. One Orco-QF2 transgenic line drove expression in only a subset of olfactory neurons, likely to be due to genomic silencing of gene expression ${ }^{42,43}$, and was not characterized further. The other two Orco-QF2 lines produced highly similar expression patterns. Both QUAS-mCD8:GFP transgenic lines drove similarly robust QF2-dependent reporter expression. For the work presented here, a single Orco-QF2 and a single QUAS-mCD8:GFP line were used. It should be noted that we did observe weak, uninduced expression from both QUAS reporters in a small subset of mushroom body and optic lobe neurons in adult brains, as well as weak expression in three cells in the larval maxillary palps (Supplementary Fig. 2). The nonspecific reporter expression did not interfere with the analyses of Orco + olfactory neurons.

Transgenic expression pattern in larvae and adults. We examined the expression pattern of the Orco-QF2 driver line by crossing it to the QUAS-mCD8:GFP reporter. Transgenically driven GFP expression was robust and allowed direct visualization of GFP-labelled cell bodies and sensilla in larvae (Supplementary Fig. 3) and in adult mosquitoes without immunostaining (Fig. 1a,b and Supplementary Fig. 4). GFP fluorescence was evident in the antennae and maxillary palps of third and fourth instar larvae (Supplementary Fig. 3), and in the antennae, maxillary palps and proboscis of adult animals (Fig. 1). Larval antennal (female: median $=10$ cells per antenna, interquartile range $(\mathrm{IQR})=2$ cells per antenna, $n=20$; male: median $=11$ cells per antenna, $\mathrm{IQR}=1.75$ cells per antenna, $n=14$ ) and maxillary palp (both female and male: median $=5$ cells per palp, IQR $=1$ cell per palp, $n=20$ ) expression was similar between males and females (Supplementary Figs 3 and 5, and Supplementary Table 1). Expression patterns in the adult antennae and maxillary palps (Fig. 1c,d) were sexually dimorphic, consistent with previous reports ${ }^{17,44}$. Approximately 500-600 cells were labelled in the female antenna, with GFP + cells and trichoid sensilla densely distributed in the 12 distal antennal segments (Fig. 1a,c). In contrast, $\sim 230 \mathrm{GFP}+$ cells, located predominantly in the distal 2 segments, were detectable in the male antenna (Fig. 1b,d). Previous studies report that $95 \%$ of trichoid sensilla contain two olfactory neurons ${ }^{13}$. When combined with trichoid sensilla counts $(\sim 630$ trichoid sensilla in females and $\sim 225$ in males $)^{15,45,46}$, this predicts $\sim 1,250$ olfactory neurons in female antennae and $\sim 550$ olfactory neurons in male antennae. Interestingly, electron microscopy studies of A. stephensi antennae report five different types of trichoid sensilla based on physical characteristics ${ }^{15,46}$. Given the numbers of Orco + neurons identified here, this suggests that many types of trichoid sensilla may not be innervated by Orco + neurons, but may be innervated by other classes of olfactory neurons. It could also be possible that some or all trichoid sensilla contain one Orco + and one Orco - olfactory neuron, although this arrangement is rarely observed in Drosophila.

The maxillary palps in A. gambiae have $\sim 67$ capitate pegs in females and $\sim 14$ pegs in males ${ }^{15}$. We observed $120 \mathrm{GFP}+$ cells in female maxillary palp capitate pegs (Fig. 1c,d) distributed along segments 2-4 of the palp (Fig. 1a,c) and $40 \mathrm{GFP}+$ cells in the male maxillary palp sparsely distributed in the distal club (Fig. 1b,d). These numbers suggest that approximately two cells are labelled per capitate peg, in agreement with previous reports ${ }^{22}$ that two Orco-dependent ORs (OR8 and OR28) are expressed in the maxillary palps. Expression in the proboscis shows a similar pattern in males and females, with $\sim 45 \mathrm{GFP}+$ cells located in the labella of the proboscis (Fig. 1c,d).

There were no GFP-labelled cells besides the antennae and maxillary palps in third to fourth instar larvae. In adults, we focused our investigation only on external tissues for Orco-QF2, QUAS-mCD8GFP expression, which we found was limited to the expression in the olfactory organs detailed above. It remains to be determined whether other tissues and cell types, such as sperm ${ }^{47}$, are also labelled by Orco-QF2.

Validation of expression. To verify that the Orco-QF2 driver accurately targets Orco-expressing neurons, we immunostained peripheral appendages of Orco-QF2, QUAS-CD8:GFP animals with antibodies directed against DmOrco ${ }^{29}$ and CD8 (Fig. 2 and Supplementary Fig. 6), comparing immunofluorescence signals in these two channels with GFP fluorescence. Whole-mount antibody staining of olfactory tissues can be challenging due to limited access to neurons within cuticular tissues. Anti-CD8 acted as a control for the efficiency of antibody labelling of CD8:GFP protein and antibody staining was often less effective than labelling observed from native GFP fluorescence. In larval antennae and maxillary palps (Supplementary Fig. 6) 91-100\% of Orco + cells were labelled by the anti-CD8 antibody, whereas $94-100 \%$ of CD8 + cells were also labelled by the anti-DmOrco antibody (Supplementary Table 2). Similarly, in the adult antennae, maxillary palps and labella, the overlap between antiOrco and anti-CD8 staining included $100 \%$ of Orco + cells and 84-97\% of CD8 + cells (Fig. 2 and Supplementary Table 3). We also performed sections of adult antennae and immunostained for anti-DmOrco (Supplementary Fig. 7). We found 100\% of antiDmOrco + sensilla were co-labelled by GFP (201 sensilla in 19 samples).

These results indicate that the Orco-QF2 line robustly labels cells that are detectable with anti-DmOrco and also a small number of cells not detectable by anti-DmOrco. It is possible that these extra cells may represent Orco- olfactory neurons. Alternatively, as binary expression systems allow for amplification of weak promoter signals, it is plausible that the QUAS$m C D 8: G F P$ reporter labels neurons that express low levels of Orco protein and are not detectable by the anti-DmOrco staining due to the background of this antibody when used in Anopheles tissues.

Orco + neuronal targets in the adult mosquito brain. Using our neurogenetic approach we find that olfactory receptor neurons labelled by the Orco-QF2 driver line send their axons to two distinct areas of the brain: the AL and the SEZ (Fig. 3a). The $\mathrm{AL}$ is larger and has denser Orco + neuron innervation in 
a

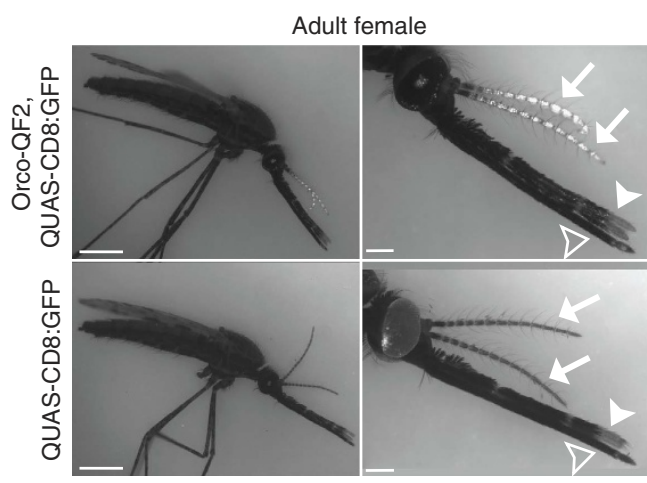

C

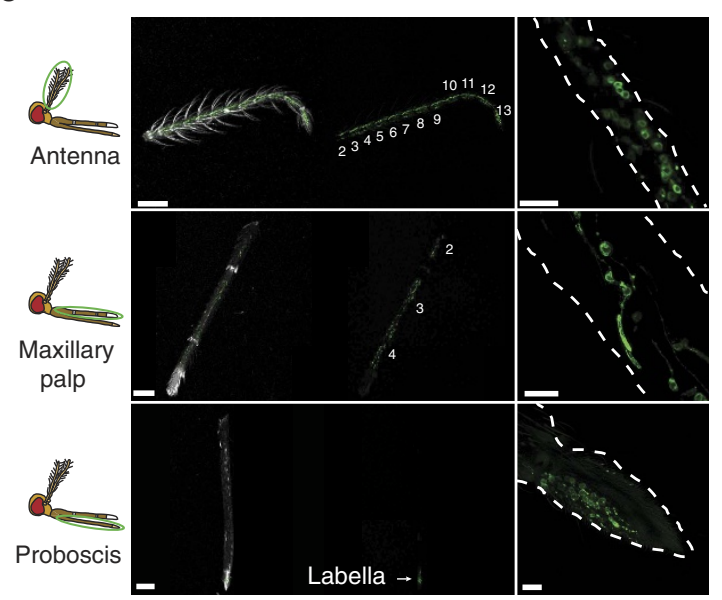

b

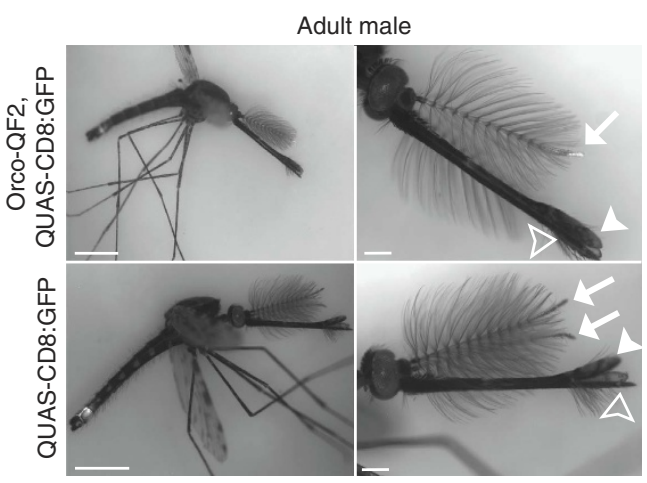

d

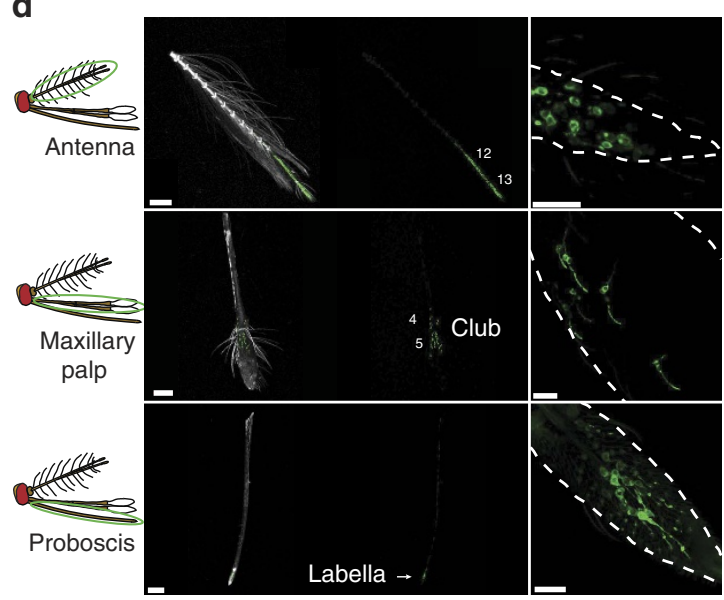

Figure 1 | Orco-QF2 expression in olfactory neurons in adult. (a,b) Membrane-targeted GFP expression in adult female (a, top row) and male (b, top row) animals. Bottom row shows images of female (a) and male (b) QUAS-mCD8:GFP controls. GFP expression is visible in antennae (arrows), maxillary palps (white arrowheads) and proboscis (open arrowheads) of transgenic animals (top row), but not in QUAS-mCD8:GFP controls (bottom row). Transgenic animals also express fluorescent DsRed and/or CFP markers in the eye, which results in residual fluorescence in the GFP channel. Genotype: Orco-QF2, QUAS-mCD8:GFP. Scale bars, (a,b) left panels, $1 \mathrm{~mm} ;(\mathbf{a}, \mathbf{b})$ right panels, $200 \mu \mathrm{m}$. (c,d) Confocal images of GFP expression in the antennae (c,d, top rows), maxillary palps (c,d, middle rows) and proboscis (c,d, bottom rows) of female (c) and male (d) adult animals. Images in $\mathbf{c}, \mathbf{d}$ left panels were acquired in RFP (autofluorescence) and GFP channels, which are shown separately for clarity. (c,d) Right panels are high-magnification images showing the cell bodies of labelled neurons. Scale bars, (c,d) left panels, $200 \mu \mathrm{m}$; (c,d) right panels, $20 \mu \mathrm{m}$.

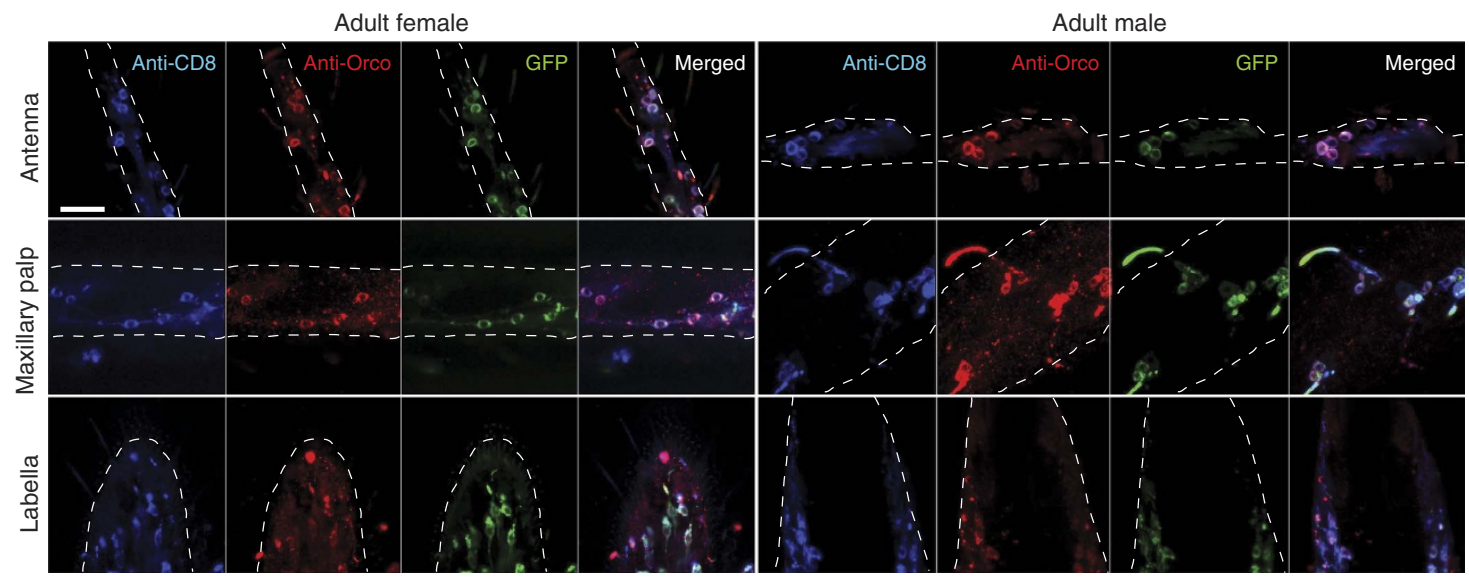

Figure 2 | Orco-QF2 drives GFP reporter expression in adult Orco + neurons. Antennae (top row), maxillary palps (middle row) and proboscis (bottom row) of adult female (left) and male (right) mosquitoes were immunostained with anti-CD8 (blue) and anti-DmOrco (red) antibodies. Images show immunofluorescence of CD8 and Orco, as well as genetically driven GFP (green). Same cell bodies are labelled in all three channels. White dashed line marks borders of the imaged tissue. All images were acquired the same magnification. Genotype: Orco-QF2, QUAS-mCD8:GFP. Scale bar, 20 $\mu$ m. 
a

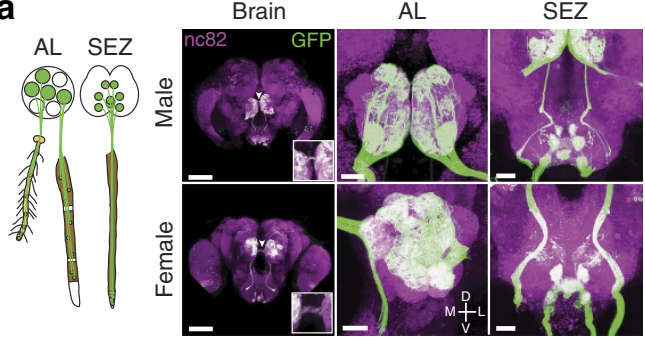

b

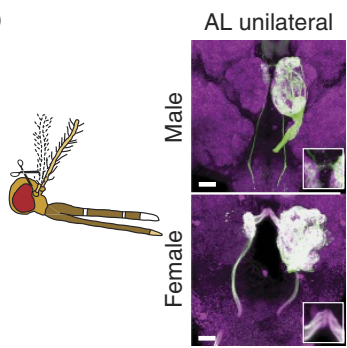

C Antennal backfill

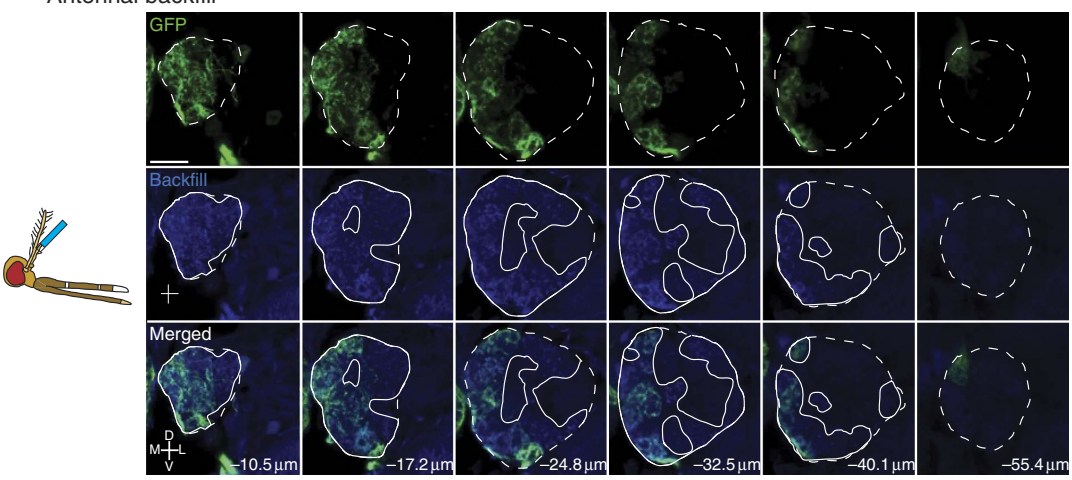

d

Maxillary palp backfill

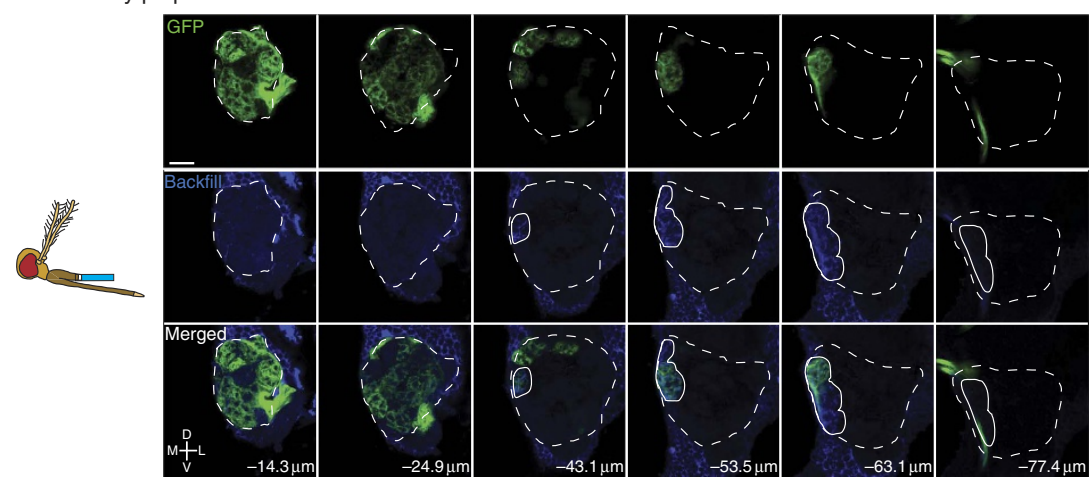

e

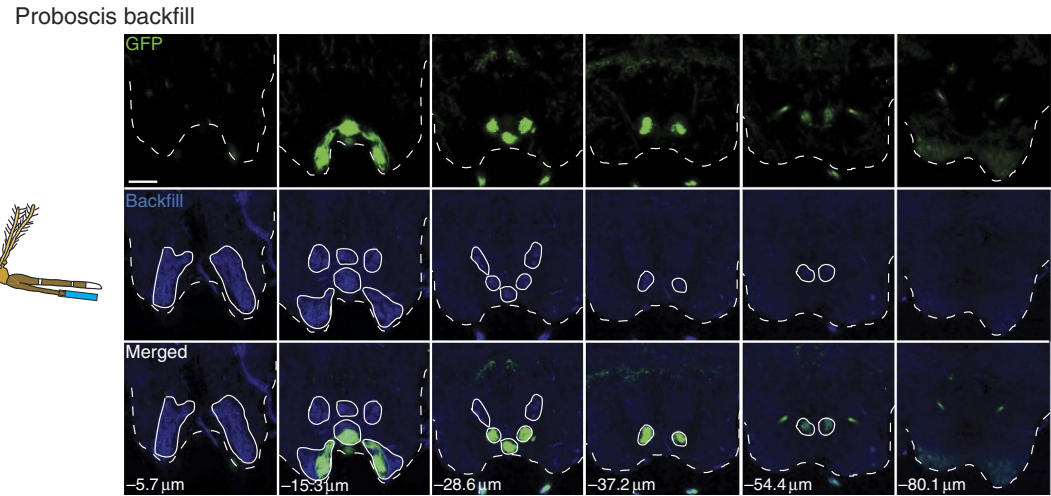

Figure 3 | Orco + olfactory neurons target two sensory brain regions. (a) Orco + receptor neurons send projections to the AL and SEZ of the brain, as detected in Orco-QF2, QUAS-mCD8:GFP animals by anti-GFP antibody labelling. Left column shows an overall image of the brain (scale bar, $100 \mu \mathrm{m}$ ). Middle and right columns show the AL and SEZ at higher magnifications (scale bar, $20 \mu \mathrm{m}$ ). Confocal sections are maximum z-projections. Arrowhead points to commissure. Inset shows commissure at higher magnification. (b) Shown are brains of male and female Orco-QF2, QUAS-mCD8:GFP mosquitoes in which the right antennae had been ablated (as schematized in the cartoon) 5 days before brain dissections. The ALs retain ipsilateral Orco + innervation from the unablated left antennae and bilateral innervation from the intact maxillary palps. Inset shows commissure at higher magnification. Scale bars, $20 \mu \mathrm{m}$ (c-e). Antennae (c), maxillary palps (d) or proboscis (e) of Orco-QF2, QUAS-mCD8:GFP mosquitoes were cut to $\sim 1 / 3$ of their length (as depicted in the cartoons) and backfilled by neurobiotin that is incorporated into the membranes of severed neurons. The neurobiotin labelling (blue) of the brains, together with the GFP labelling (green), establishes the origin of Orco + receptor neurons (antennal, maxillary palp or proboscis) that innervate the AL and SEZ. Backfills originating from the antenna innervated only the ipsilateral AL. Backfills originating from the maxillary palps innerved both ipsilateral and contralateral ALs. Backfills originating from the proboscis innervated only the SEZ brain region. The numbers indicate the distance in micrometres from the most anterior confocal section. Dashed white lines outline the AL/SEZ. Solid white lines outline the area labelled by the neurobiotin backfill (blue signal). Scale bars, $20 \mu \mathrm{m}$. 
females than in males (Fig. 3a). To quantify these differences, we performed three-dimensional (3D) modelling of male and female A. gambiae brains and antennal lobes (Methods). Female brains $\left(5.1 \times 10^{6} \pm 0.3 \times 10^{6} \mu \mathrm{m}^{3}\right.$, mean \pm s.e.m., $\left.n=3\right)$ were 1.07 times larger in volume than male brains $\left(4.8 \times 10^{6} \pm 0.3 \times 10^{6} \mu \mathrm{m}^{3}\right.$, mean \pm s.e.m., $n=3$ ). In contrast, female antennal lobes $\left(1.7 \times 10^{5} \pm 0.1 \times 10^{5} \mu \mathrm{m}^{3}\right.$, mean \pm s.e.m., $\left.n=5\right)$ were 1.9 times larger than male antennal lobes $\left(0.9 \times 10^{5} \pm 0.02 \times 10^{5} \mu \mathrm{m}^{3}\right.$, mean \pm s.e.m., $n=3$ ), reflecting increases in individual glomerular volumes. The increase in $\mathrm{AL}$ size in females is in agreement with a larger number of Orco + receptor neurons in the antennae and maxillary palps innervating AL glomeruli. Some Orco + neurons project bilaterally, as evidenced by a clearly labelled commissure between the left and right ALs (Fig. 3a,b). Unexpectedly, the SEZ area of the brain is also innervated by Orco + neurons in a pattern of six strongly labelled and two weakly labelled glomerular structures (Fig. 3a). As in Drosophila the SEZ region is known to receive gustatory inputs from the proboscis, we hypothesized that $\mathrm{Orco}+$ neurons from the mosquito labellum projected to the SEZ.

To determine the origin of the bilaterally projecting Orco + neurons, we ablated the right antennae of adult Orco-QF2, QUAS$m C D 8$ :GFP animals and maintained them for 5 days in standard conditions, to allow sufficient time for the axons of the severed olfactory neurons to degenerate, as previously demonstrated in Drosophila ${ }^{48}$. The ALs retained Orco + commissural labelling and innervation of only two posterior glomeruli in the right (ipsilateral) AL of the brain, whereas the left AL remained largely unaffected (Fig. 3b). This suggests that antennal olfactory neurons project only to the ipsilateral $\mathrm{AL}$, consistent with previous results $^{49,50}$ and in contrast to bilateral projection patterns of Drosophila antennal olfactory neurons ${ }^{48}$. The two remaining glomerular targets probably originate from the maxillary palp. To verify and extend these results, we attempted to label severed olfactory neurons from the different olfactory tissues with the anterograde tracing dye neurobiotin ${ }^{51}$. The neurobiotin backfill labelling of severed antennal (Fig. 3c) and maxillary palp (Fig. 3d) neurons indicated that the two bilaterally projecting glomeruli are indeed innervated by maxillary palp neurons. Thus, all antennal Orco + neurons project exclusively to the ipsilateral side of the brain, whereas maxillary palp Orco + neurons innervate the AL bilaterally, consistent with previous backfill studies ${ }^{49,51}$. Neurobiotin backfills of antennae and maxillary palps resulted in staining of all but three posterior glomeruli that were not reliably labelled in every examined brain (see Fig. 4 for details); however, the antennal and maxillary palp backfills did not stain the SEZ. Neurobiotin backfills of the proboscis (Fig. 3e) confirmed that the SEZ is innervated by Orco + neurons from the labella. In contrast to a previous report ${ }^{52}$, we observed no neurobiotin + or GFP + projections from the proboscis to the AL.

Olfactory neuron innervation model of the AL. Using our neurogenetic approach we identified 67-70 glomeruli in the AL of females and 67-68 glomeruli in the AL of males (Supplementary Table 4). In contrast to Drosophila, the appearance of the AL in A. gambiae varies markedly among animals (see Methods), which could explain the slight variation in the number of glomeruli we counted. Previous studies ${ }^{51}$ reported 60 glomeruli in a female and 61 glomeruli in a male AL, and assigned names to the glomeruli based on their anatomical position. However, previous work assumed that a large part of the anterior AL was occupied by projections from Johnston's organ mechanosensory neurons ${ }^{51}$. This contradicts the transgenic Orco $>$ GFP expression we observe, in which the majority of the anterior glomeruli are
Orco + . Furthermore, we did not observe GFP expression in the Johnston's organ, consistent with a previous study showing a lack of Orco immunoreactivity in this tissue ${ }^{17}$. All together, this suggests that these anterior AL glomeruli do not constitute a mechanosensory centre. To avoid confusion, we have renamed the glomeruli, assigning number 1 to the most dorsal and anterior glomerulus and subsequent numbers to more ventral and posterior glomeruli (Fig. 4a and Supplementary Table 5). We also have made available a $3 \mathrm{D}$ reconstruction model of these data for future studies, to allow renaming of these glomerular regions based on specific OR identities (see Methods).

Transgenic GFP expression, combined with the neurobiotin backfill experiments, allowed us to assign each AL glomerulus to one of four classes based on the origin of their respective receptor neurons (antennal or maxillary palp) and on the status of Orco-QF2, QUAS-mCD8:GFP labelling (Fig. 4a). We identified 33 Orco + glomeruli in both females and males (Supplementary Table 5), despite the $\sim 2.5$-fold sexually dimorphic difference in the number of Orco + receptor neurons. The majority of the remaining $\sim 50 \%$ of glomeruli are likely to be innervated by olfactory neurons that express either an IR or GR receptor.

Five glomeruli-2 Orco + (numbered 45 and 55, blue colour in Fig. 4a) and 3 Orco - (numbered 62, 63 and 64, yellow colour in Fig. 4a)-were reliably labelled by maxillary palp backfills. The two Orco + glomeruli probably correspond to the OR8 and OR28 receptor neurons ${ }^{22}$, and at least one of the three Orcoglomeruli are innervated by neurons expressing the $\mathrm{CO}_{2}$-sensing GR22-GR23-GR24 receptor complex ${ }^{22}$.

Varying numbers of glomeruli were labelled by antennal backfills (purple and green colours in Fig. 4a), with 3 posterior glomeruli (numbered as 51, 52 and 67, grey colour in Fig. 4a) exhibiting unreliable labelling. This variability in labelling may be explained by weak labelling of posterior glomeruli and also by the fact that neurons originating in the proximal $25-30 \%$ of antennae were not labelled in the backfill experiments and may be projecting to these unlabelled glomeruli. This ambiguity will be resolved by future transgenic labelling of neurons that express single receptor types.

The ability to distinguish the olfactory tissue of origin and the Orco-expression status for glomerular targets allowed us to predict a functional map of the AL (Fig. 4b). The organization of glomeruli within the AL is remarkably similar between Drosophila and A. gambiae (Fig. 4b). Orco + glomeruli are anterior, whereas Orco - glomeruli (IR and GR glomeruli in Drosophila) are posterior. This potential evolutionary conservation of AL anatomy suggests that in A. gambiae, as in Drosophila, most posterior Orco- glomeruli are targeted by IR-expressing neurons. The ventral location of GR + glomerular targets in Drosophila is suspected to have coincided with a developmental change during which GR + neurons located in the maxillary palps switched to the antennae $e^{53}$. Thus, it is likely to be that the medial-central glomerular location of Orco - , MP + glomeruli in Anopheles reflects tissue of origin, as all maxillary palp olfactory neurons in Drosophila also target medial-central glomeruli $^{9,10}$. It remains to be explored whether the similarities in olfactory neuronal organization between the two species are also seen in higher brain regions ${ }^{54}$.

Olfactory innervation model of the SEZ. In the SEZ, we identified eight glomerular structures formed by Orco + neurons originating from the labellum (Fig. 4c). Males and females exhibited similar SEZ innervation patterns. From these confocal images, we generated a $3 \mathrm{D}$ reconstruction of the Orco + olfactory neuron innervation pattern in the SEZ and outlined the glomeruli in different colours within the olfactory nerve tracts (Fig. 4d). 
a

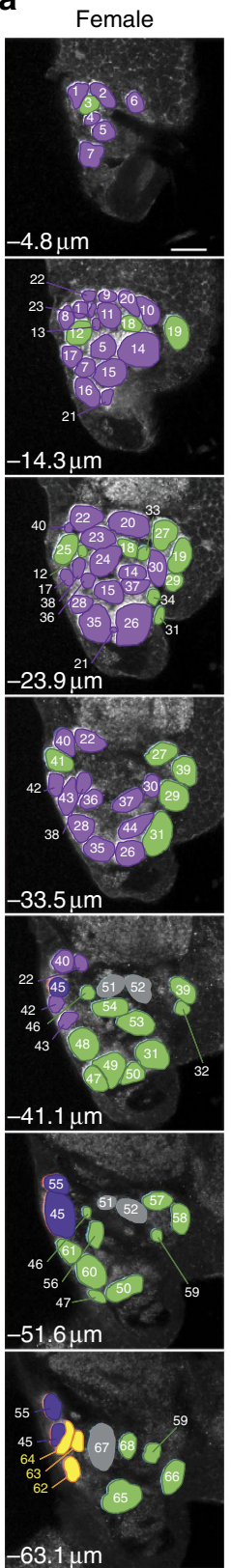

Orco+, Antenna+

Orco-, Antenna+
Male
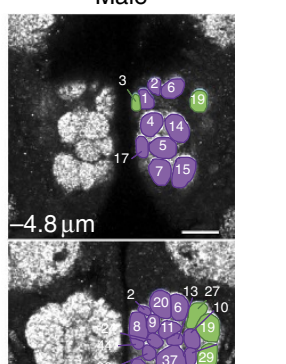

b
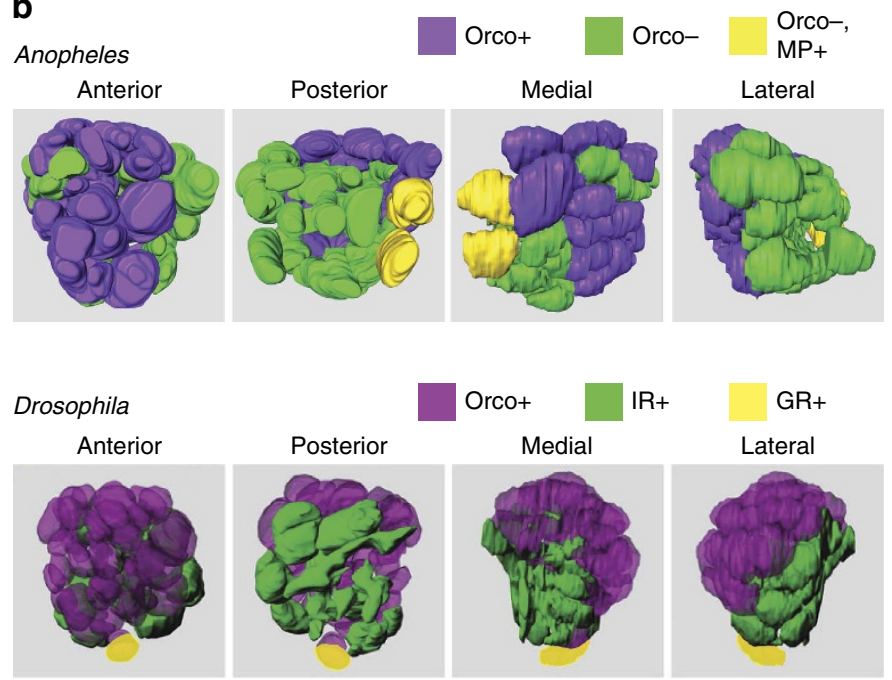

C
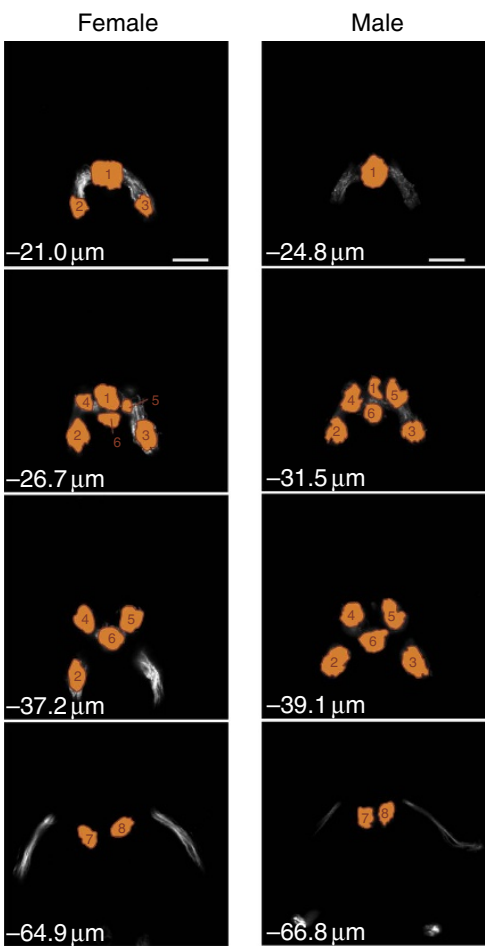

Orco+, labellum+ d

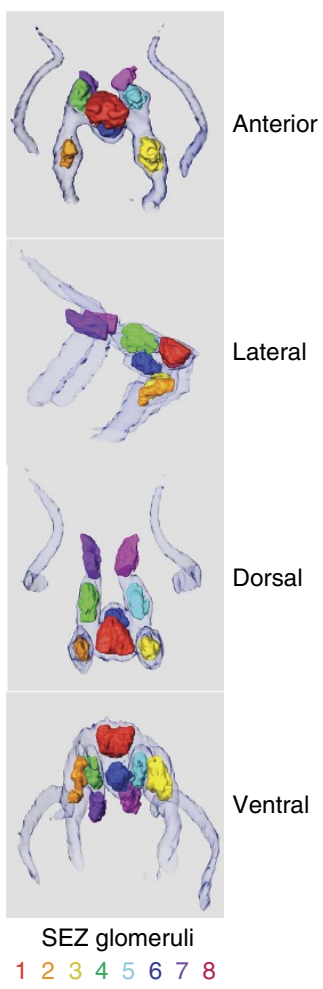

Not labelled reliably by backfills

Figure 4 | Reconstruction of the mosquito AL and SEZ based on Orco expression and olfactory tissue of origin. (a) Confocal Z-stacks of female (left) and male (right) ALs, shown at the same magnification (most anterior-top row, most posterior-bottom row). Glomeruli were outlined manually, and GFP and neurobiotin backfill signals were used to assign each glomerulus to one of five groups: Orco + antennal glomeruli (purple), Orco - antennal glomeruli (green), Orco + maxillary palp glomeruli (blue), Orco - maxillary palp glomeruli (yellow) and glomeruli that were not labelled by backfills (grey). Glomeruli were numbered starting at the most anterior section. Scale bars, $20 \mu \mathrm{m}$. Also see Supplementary Table 5. (b) A. gambiae and D. melanogaster ALs show similarities in the arrangement of glomeruli targeted by Orco + (purple) and Orco - neurons. Orco - neurons originating from the mosquito antennae are likely to be IR-expressing neurons (IR, green) and Orco - neurons from the maxillary palps are likely to be GR-expressing neurons (GR, yellow) ${ }^{22}$. The Drosophila AL is reprinted with permission from ref. 76 with minor modifications. (c) Confocal Z-stacks of female (left) and male (right) SEZ, shown at the same magnification (most anterior, top row; most posterior, bottom row). Glomerular-like structures were outlined manually using the GFP signal. Glomerular structures were numbered starting at the most anterior section. Scale bars, $20 \mu \mathrm{m}$. (d) Three-dimensional modelling of olfactory neuron targeting in the SEZ. The Orco-QF2-labelled nerve bundle from the labella is shown for reference.

This highlights the relative positions of the eight labeller glomeruli, with three symmetrical pairs of lateral glomeruli and two central glomeruli along the SEZ midline (see Methods for availability of source data). Backfill experiments of the proboscis (Fig. 3) labelled these glomerular structures and also a broader region in the SEZ probably innervated by gustatory and 
mechanosensory neurons ${ }^{52,55,56}$ (see Methods for availability of source data). As the labellum is in close proximity to host tissue during bloodfeeding ${ }^{15}$, this raises the possibility that it functions to integrate sensory signals from host odorants and tastants.

\section{Discussion}

We report here the first neurogenetic labelling of olfactory neurons in mosquitoes. By visualizing the entire neuronal processes of Orco + olfactory receptor neurons, these studies revealed that two brain regions are targeted by olfactory receptor neurons in this species-the AL and the SEZ. This is the first time, to our knowledge, that insect olfactory receptor neurons have been found to innervate brain regions outside the AL. These Orco + neurons originate in the labella, a structure that is in close proximity to host skin during bloodfeeding, suggesting that volatiles of the skin or in the blood may activate these olfactory receptor neurons in female mosquitoes. Indeed, recordings from T2 sensilla on the labella indicate activation by low volatility odorants often found on host tissues or in blood 52,57 .

The labellum also contains gustatory neurons ${ }^{52,55,56}$ that in the Drosophila proboscis are known to innervate the $\mathrm{SEZ}^{23}$. It is possible that this region in the mosquito SEZ is a sensory integration centre combining olfactory and gustatory signals. In humans, the sensory integration of taste and smell gives rise to a perception of flavor. Whether this region in the mosquito SEZ underlies a 'flavor' centre remains to be explored. Interestingly, Orco + olfactory neurons were recently identified at the tip of the hawkmoth (Manduca sexta) proboscis and found to be involved in evaluating the quality of flowering plants during pollination ${ }^{58}$. Whether the hawkmoth Orco + proboscis neurons innervate the SEZ and thus implicate a common sensory integration centre between Lepidoptera and Diptera remains to be determined.

The ability to label the entire neuronal process was accomplished genetically by introducing the Q-binary expression system, comprising a QF2 driver line and QUAS-geneX reporter line, into A. gambiae. Binary expression systems are powerful genetic tools for investigating and manipulating neuronal circuits, and adoption of these reagents into the mosquito system should accelerate studies in neuronal processing of these dangerous insects. In this work, we used the putative AgamOrco enhancer + promoter region to capture the expression pattern of roughly half of all olfactory neurons in A. gambiae. Similar approaches could be applied to capture the expression patterns of olfactory neurons not labelled by Orco, such as the IR neurons and the $\mathrm{CO}_{2}$-sensing GR neurons. By using the promoters from the ionotropic co-receptors Ir8 or Ir25, and the promoters from one of the three GR $\mathrm{CO}_{2}$-sensing complex (Gr22, Gr23 and Gr24), it might be possible to generate new QF2 lines labelling these olfactory neuron populations. To this end, we did generate Gr24-QF2 transgenic lines using a $961 \mathrm{bp}$ putative enhancer + promoter region, but this did not yield expression. In such cases, alternative genetic strategies, pioneered in Drosophila, can be used for capturing tissue-specific expression patterns to drive QF2 (ref. 59).

There are two major advantages to using a binary expression system for tissue labelling. The first advantage is amplification of a promoter signal. If a genomic promoter is weak, meaning transcription driven by the promoter results in only few copies of the target gene, it could be difficult to visualize these tissues directly with conventional fluorescent markers. Yet, when a weak promoter expresses a transcriptional activator such as QF2, even low levels of the activator will continue to drive reporter expression. In our system, we used 15 copies of the QF-binding site QUAS, which should lead to robust amplification of even weak promoters. This is supported by the fact that membrane-targeted GFP fluorescence in olfactory neurons using the Orco promoter is visible even in live animals.

The second advantage is versatility. The Orco-QF2 line, which has been validated here, can be crossed to other QUAS-geneX effector lines for a range of studies. In our experience, all QUAS-mCD8:GFP insertions appeared to function in Anopheles, suggesting that the generation of additional QUAS-geneX lines will not be a limiting step. We chose first to generate a membrane-targeted GFP reporter, QUAS-mCD8:GFP, for efficient tissue labelling and anatomical studies. This line will also be useful when validating the expression patterns of new QF2 driver lines. Future studies could instead use reporters that monitor neuronal activity, for example, GCaMP6 (ref. 60). Such reagents will allow the visualization of olfactory neuron activity in response to odours in the peripheral organs and also in their glomerular targets in the brain. These types of studies have been used to characterize an 'olfactory code' in Drosophila $24,61,62$ and can be applied to similar studies in Anopheles, to determine the Orco + olfactory code that distinguishes the odours of humans from those of other animals. Besides monitoring neuronal activity, other QUAS effector lines can be used to manipulate or modulate neuronal activity. For example, by using QUAS-Chrimson ${ }^{63,64}$ or QUAS-OrX, olfactory neuron activities could be modulated by red light or odorants, respectively. This approach has yet to be tested in mosquitoes, but it has the potential to provide insights into effective strategies that disrupt olfactory-based host seeking. A rich repertoire of genetic tools developed in Drosophila can be adopted for similar uses in mosquitoes $^{36}$.

We have generated a map of the adult $A$. gambiae AL. We have identified glomeruli that are targeted by the antennal or maxillary palp neurons and glomeruli that are targeted by Orco + or Orco - olfactory receptor neurons. This allowed us to predict which glomeruli may be targeted by IR neurons (Orco-, antennal) and which glomeruli may be targeted by the $\mathrm{CO}_{2}-$ sensing neurons (Orco-, maxillary palp). A previous study speculated that a large portion of the A. gambiae AL comprised mechanosensory targets from Johnston's organ in the pedicel ${ }^{51}$. However, given that these same glomeruli are clearly innervated by Orco + olfactory neurons, this seems unlikely. Instead, the AL structure is primarily innervated by olfactory neurons from the antennae and maxillary palps. As in Drosophila ${ }^{65}$, the mosquito antennae may contain sensory sensilla that respond to heat and whose neurons target posterior Orco - $\mathrm{AL}$ glomeruli ${ }^{66}$.

By speculating which glomeruli are targeted by Orco + receptor neurons, IR neurons and GR neurons, the Anopheles AL map presented here also highlights the glomeruli likely to be activated by different types of odorants. ORs tend to be activated by host body odorants, such as 4-methylphenol, indole or 1-octen-3-ol ${ }^{11,25}$, whereas IRs may be activated by acids and amines found in host body odours, such as lactic acid and ammonia $^{18}$. The GR neurons will be activated primarily by carbon dioxide ${ }^{22,67}$. The number of glomeruli innervated by Orco + receptor neurons and IR neurons is roughly equivalent, suggesting that host seeking behaviours may rely equally on both types of olfactory neuron signalling. This is consistent with studies in A. aegypti in which host seeking was not abolished in Orco mutant mosquitoes ${ }^{68}$.

The number of Orco + glomeruli in the male and female ALs was the same, despite the sexually dimorphic location and number of Orco + neurons in the antennae and maxillary palps. A notable sexually dimorphic difference was the reduced size of male glomeruli and ALs: $\sim 1.9$-fold smaller in volume in males compared with females. This probably reflects the reduced number of innervating olfactory neurons in the glomerular circuit. If each female Orco + glomerulus can be matched to a 
male Orco + glomerulus and each glomerulus is innervated by neurons expressing the same olfactory receptor(s), this suggests the male olfactory system may represent a scaled-down version of the female olfactory system. As such, the male has the potential to respond to all the same odorants that the female responds to, but due to the reduced number of olfactory neurons, with reduced acuity. This is in agreement with transcriptome profiling of the antennae between males and females ${ }^{45}$. When sensillar numbers were normalized between the sexes, the identity and expression levels of ORs between males and females were remarkably similar $^{45}$. The transcriptome study identified 59 AgORs enriched at least twofold in the antennae and maxillary palps (compared with the body $)^{45}$. In contrast, we were able to identify only 33 glomeruli innervated by Orco + neurons. It is possible that glomeruli in our model might be further subdivided into additional glomeruli based on specific OR innervations. Another possibility is that mosquito olfactory neurons may frequently express more than one $\mathrm{OR}^{69,70}$. In particular, Anopheles OR genes clustered in the genome (within $\sim 450 \mathrm{bp}$ of each other) are often co-expressed in the same olfactory neuron (for example, AgOR13, AgOR16, AgOR17 and $A g O R 55)^{69,70}$. Including all $\mathrm{Ag}$ OR gene clusters (VectorBase, AgamP4), as many as $17 \mathrm{Ag}$ OR genes might be co-expressed in as few as 6 olfactory neuron types. Furthermore, if receptor combinations are sexually dimorphic for each neuron, this could be a mechanism to enable differential response profiles between males and females, while maintaining overall expression of the same set of ORs.

The maxillary palp is a simplified olfactory organ, containing only capitate peg sensilla that house olfactory neurons. Prior electrophysiological studies identified three neurons within each capitate peg sensillum, with two of these neurons expressing Orco and the third expressing the Gr22-Gr23-Gr24 receptor complex and being $\mathrm{CO}_{2}$ sensitive ${ }^{22}$. By ablating one antenna and allowing for the Orco + olfactory neurons in the antenna to degenerate, we visualized the two glomerular targets for the two Orco + olfactory neurons originating from the maxillary palp capitate peg sensilla. By using neurobiotin backfills of severed maxillary palp nerves, we identified five posterior glomeruli in the AL that were innervated from the maxillary palp, including the two glomeruli innervated by the Orco + olfactory neurons. These data suggest that Orco - neurons from the maxillary palp innervate three glomeruli. The capitate peg Gr22-Gr23-Gr24 $\mathrm{CO}_{2}$-sensing neuron must innervate at least one of these three glomeruli. The other two glomeruli will either be innervated by the $\mathrm{CO}_{2}$-sensing olfactory neurons or currently unidentified nonchemosensory neurons. Besides the capitate pegs, the maxillary palp contains two other innervated sensilla-the campaniform sensilla and sensilla chaetica that probably function as mechanoreceptors, proprioreceptors or touch sensors ${ }^{14,15}$. Alternatively, some of these Orco - glomeruli may be innervated by neurons in uncharacterized sensilla that respond to non-mechanical stimuli such as humidity or temperature, as found in the Drosophila $\mathrm{AL}^{71}$.

We also generated the first map of the Orco + olfactory receptor neuron targets in the A. gambiae SEZ. These may represent a previously unreported mode of olfactory signalling independent of the AL in an insect brain. In Drosophila, gustatory neuron targeting in the SEZ is diffuse ${ }^{23}$. Interestingly, the Orco + olfactory neurons in A. gambiae appear to form eight glomerular structures in the SEZ region. A number of questions regarding this innervation remain to be explored. Are these olfactory neuron targets also innervated by gustatory neurons? Do all labellar Orco + olfactory neurons innervate all SEZ glomeruli or do subsets exist based on OR expression? Are these olfactory glomeruli organized to influence gustatory signals such that favourable host odours trigger pleasant tastes and unfavourable host odours trigger bitter-like tastes?

As vectors for viral and parasitic diseases, anthropophilic insects pose a major threat to human health. Mosquitoes are particularly dangerous due to their ability to visit multiple people during a bloodmeal, such that infections can rapidly spread through both human and mosquito populations. Effectively controlling the spread of mosquito-borne diseases will probably require a multitude of approaches. Given that each step in host seeking-from long-range guidance to short-range bitinginvolves cues from the olfactory system, targeting this sense could provide an effective means of reducing biting and the spread of disease. The neurogenetic tools introduced here may help identify which aspect of olfactory host-seeking, such as labellar probing or long-range guidance, is the most vulnerable to interference, and thereby guide new strategies for olfactory-based interventions.

\section{Methods}

Recombinant DNA construction. Plasmids were constructed by enzyme digestions, PCR, subcloning and the In-Fusion HD Cloning System (Clontech, catalogue number 639645). Plasmid inserts were verified by DNA sequencing.

The pXL-BACII-ECFP-15xQUAS_TATA-mCD8-GFP-SV40 construct was used to generate transgenic animals, carrying a reporter transgene QUAS$m C D 8-G F P$. The $15 x Q U A S$ sequence was PCR amplified from $p Q U A S p$ plasmid (Addgene \#46162) using oligos $p$ Bac-ECFP-15xQUAS-Inf-FOR (5'-aga gcg gcc gcc acc gcg gtc acg tgt cac tgg gtc ag- $\left.3^{\prime}\right)$ and $p B a c-E C F P-15 x Q U A S-I n f-R E V\left(5^{\prime}\right.$-tac cgt cga cct cga gct agc agg tcc tca ctg agt ccc aac gtg aaa g- $3^{\prime}$ ) and InFusion-cloned into $p X L-B a c I I-E C F P$ plasmid $^{72}$, digested with XhoI and SacII. Next, the TATA$m C D 8$-GFP-SV40-SV40 sequence was PCR amplified from $p Q U A S t-m C D 8-G F P$ plasmid $^{34}$; Addgene \#24351) using oligos pBac-ECFP-GFP-Inf-FOR (5'-act cag tga gga gga cct gaa ttc ctg cag ccc g- $\left.3^{\prime}\right)$ and $p B a c-E C F P-G F P-I n f-R E V\left(5^{\prime}-\right.$ tac cgt cga cct cga gag atc tag gcc ttc tag tgg atc $\left.\mathrm{cg}-3^{\prime}\right)$, and InFusion-cloned into the intermediate step construct, obtained at the previous step, that was digested with PpuMI and XhoI.

The pXL-BacII-15xQUAS_TATA-SV40 construct may be used to easily generate new QUAS reporter plasmids by sub-cloning a reporter gene into the multi-cloning site between TATA promoter and SV40 terminator sequences. To create $p X L-B a c I I-15 x Q U A S \_T A T A-S V 40, p X L-B A C I I-E C F P-15 x Q U A S \_T A T A-$ $m C D 8-G F P-S V 40$ was digested with XhoI and EcoRV-HF to remove the CD8GFP-SV40-SV40 cassette. The SV40 terminator was PCR amplified from $p Q U A S t$ $m C D 8$-GFP (ref. 34; Addgene \#24351) with oligos pBac-15xQUAS-SV40-Inf-FOR $\left(5^{\prime}\right.$-gcg gec gcg get cga gac gtc gat ctt tgt gaa gga acc tta ctt ctg- $\left.3^{\prime}\right)$ and $p B a c-$ 15xQUAS-SV40-Inf-REV $\left(5^{\prime}-\mathrm{ttt} \mathrm{ctt}\right.$ gtt ata gat atc gat cca gac atg ata aga tat gat gag- $3^{\prime}$ ) and InFusion-subcloned back into the construct.

The pXL-BACII-DsRed-QF2-hsp70 construct may be used to easily generate new QF2 (ref. 44) driver plasmids by sub-cloning an enhancer/promoter sequence upstream of QF2. To create $p X L-B A C I I-D s R e d-Q F 2-h s p 70$, the QF2-hsp70 cassette was PCR amplified from pattB-syn-QF2-hsp70 (ref. 35; Addgene \#46115) with oligos $p B A C-D S r e d-Q F 2-I n f-F O R\left(5^{\prime}\right.$-atc aat gta tct cga ggc cgg cca aca tgc cac cca ag- $\left.3^{\prime}\right)$ and $p B A C-D S r e d-Q F 2-I n f-R E V\left(5^{\prime}\right.$-ttt ctt gtt ata gat atc gga tct aaa cga gtt ttt aag $\left.c-3^{\prime}\right)$ and InFusion-cloned into $p X L-B A C I I-D S r e d^{73}$, digested with XhoI and EcoRV-HF

The pXL-BACII-DsRed-OR7_9kbProm-QF2-hsp70 construct was used to generate transgenic animals, carrying an Orco-QF2 driver construct. A 9,312 bp sequence immediately upstream of AGAP002560 (OR7) gene was PCR amplified with oligos Agamb-OR7-Inf-FOR9_1 (5'-atc at gta tct cga gtg atg caa att gtt cgg aag aat tg- $\left.3^{\prime}\right)$ and AgambOR7-Inf-Rev1 ( $5^{\prime}$-tgg gtg gca tgt tgg ccg gcc tgc gaa cgg gaa gtg aac- $3^{\prime}$ ) from genomic DNA, extracted with the DNAeasy Blood \& Tissue Kit (Qiagen, \#69506) from the Keele strain of A. gambiae. The PCR product was InFusion cloned into the $p X L-B A C I I-D s R e d-Q F 2-h s p 70$ vector, digested with XhoI and FseI.

A. gambiae transgenics. The A.gambiae M-form strain Ngousso (the M-form of A. gambiae is now referred to as Anopheles coluzzii) were grown at $28^{\circ} \mathrm{C}, 70-75 \%$ relative humidity, $12 \mathrm{~h}$ light/dark cycle. Freshly deposited eggs were collected by providing mated, gravid females with wet filter paper as an oviposition substrate for 15-20 min, after which the eggs were collected and systematically arranged sideby-side on a double-sided tape fixed to a coverslip. Aligned embryos were covered with halocarbon oil (Sigma, series 27) and injected at their posterior pole with an injection cocktail between 30-40 min after egg laying. Injection cocktails consisted of a mixture of two plasmids, one with a piggyBac vector carrying the transgene of interest with a dominant visible marker gene, DsRed or enhanced cyan fluorescent protein (ECFP), under the regulatory control of the $3 \mathrm{xP} 3$ promoter, and a piggyBac transposase-expressing plasmid consisting of the transposase open reading frame under the regulatory control of the promoter from the A. stephensi vasa gene. 


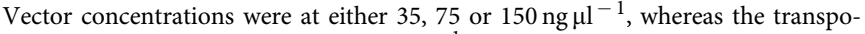
sase-expressing plasmid was at $300 \mathrm{ng} \mu^{-1}$ in $5 \mathrm{mM} \mathrm{KCl}, 0.1 \mathrm{mM}$ sodium phosphate $\mathrm{pH}$ 6.8. Halocarbon oil was immediately removed and coverslips with injected embryos were placed in trays of water at $28^{\circ} \mathrm{C}$, where the first instar larvae hatched $\sim 24 \mathrm{~h}$ later. The Insect Transformation Facility (https://www.ibbr.umd.edu/facilities/itf) within the University of Maryland College Park's Institute for Bioscience and Biotechnology Institute performed all embryo microinjections. Adults developing from injected embryos were separated by sex before mating and small groups of five to ten injected adult males and females were mixed with wildtype Ngousso adults of the opposite sex. The progeny from these matings were screened during the third or fourth larval instar for the presence of vector-specific marker gene expression. Transgenic larvae were saved and as adults were backcrossed to Ngousso (wild type).

Using the strategy of promoter cloning as described for Orco, we also generated promoter-QF2 transgenic Anopheles using predicted promoter sequences for GR24 (AGAP001915; $957 \mathrm{bp),} \mathrm{elav} \mathrm{(AGAP001883;} \mathrm{6,569} \mathrm{bp)} \mathrm{and} n$-syb (AGAP005507; 10,194 bp). These $Q F 2$ transgenic lines did not show expression when crossed to QUAS- $m C D 8$ :GFP, suggesting the promoter fragments used were insufficient for capturing the expected expression pattern.

A. gambiae stock maintenance. A. gambiae were grown at $28^{\circ} \mathrm{C}, 70-75 \%$ relative humidity and $12 \mathrm{~h}$ light/dark cycle. Larvae were provided with TetraMin Tropical Flakes and/or Purina Cat Chow Indoor pellets ad libitum, whereas adults were provided with $10 \%$ sucrose continuously. Mated, adult females were fed human blood using temperature-controlled membrane-feeding devices or mouse blood from anaesthetized mice and eggs were collected from the resulting gravid females by providing them with a cup of water containing wet filter paper on which to deposit their eggs. Orco-QF2 and QUAS-CD8:GFP transgenes were brought together by a genetic cross and maintained in the same stock for at least seven generations with no observable reduction in the strength or breadth of GFP expression.

Immunohistochemistry. Dissection of adult brains and immunostaining were done as described previously ${ }^{74}$. In short, on day 1 heads of adult mosquitoes were fixed in $4 \%$ paraformaldehyde in $0.1 \mathrm{M}$ sodium phosphate buffer (Millonig's $\mathrm{pH}$ 7.4) with $0.25 \%$ Triton $\mathrm{X}-100$ at $4{ }^{\circ} \mathrm{C}$ for $3 \mathrm{~h}$. Next, the brains were dissected in PBS containing $0.3 \%$ Triton $\mathrm{X}-100$ (PBT), washed in PBT for $1 \mathrm{~h}$ at room temperature (RT) and permeabilized with $4 \%$ Triton X-100 $+2 \%$ normal goat serum (NGS) in PBS at $4{ }^{\circ} \mathrm{C}$ overnight. On day 2 , the brains were washed in PBT for at least $1 \mathrm{~h}$ and placed in primary antibody mix $+2 \%$ NGS in PBT for two nights at $4{ }^{\circ} \mathrm{C}$. On day 4 , the brains were washed for several hours in PBT at RT and placed in secondary antibody mix $+2 \%$ NGS in PBT for two nights at $4{ }^{\circ} \mathrm{C}$. On day 6 , the brains were washed in PBT and placed in mounting solution (Slow Fade Gold) overnight at $4{ }^{\circ} \mathrm{C}$, and mounted on a microscope slide on day 7 . To visualize CD8:GFP expression, we used rat anti-CD8 (Invitrogen \#MCD0800, 1:100) and mouse nc82 (DSHB, 1:50) primary antibodies, and Alexa-488 goat anti-rabbit (Invitrogen \#A11034, 1:200) and Cy3 goat anti-mouse (Jackson ImmunoResearch \#115-165062, 1:200) secondary antibodies.

Anterograde tracing experiments with neurobiotin were performed essentially as described in ref. 51. Live mosquitoes were cooled in a $4^{\circ} \mathrm{C}$ fridge for $10-15 \mathrm{~min}$, after which they were transferred to a petri dish and placed on ice for up to $30 \mathrm{~min}$ until they were mounted in the backfill chamber. The backfill chamber consisted of a $9 \mathrm{~cm}$ petri dish with two parallel strips of Blue-Tack (Bostik, France) on the bottom of the dish, $2 \mathrm{~cm}$ apart. Double-sided sticky tape was placed on top of one of the Blue-Tack strips. The other strip was used to hold glass pipettes, which were pulled out of glass capillaries (Harvard Apparatus, \#30-0108), and had the tips broken off to create an opening of $\sim 0.3 \mathrm{~mm}$. The pipettes were backfilled with $2 \%$ Neurobiotin (Vector Laboratories, \# SP-1155 or \#SP-1150) in $0.25 \mathrm{M} \mathrm{KCl}$. Mosquitoes were mounted on the double-sided tape and either one antenna, one maxillary palp or the proboscis was cut to $\sim 25 \%$ of the original length and inserted into the tip of the biotin-filled glass pipette. We used a separate pipette for each animal. Small pieces of damp Kimwipes were placed into the backfill chamber, to prevent desiccation of the animals. The chambers were wrapped in aluminum foil and placed at $4{ }^{\circ} \mathrm{C}$ for $20-24 \mathrm{~h}$, after which we followed the Brain immunostaining protocol, described above. When Neurobiotin-Plus (Vector Laboratories, \# SP-1150) was used for backfills, Avidin-Texas Red Conjugate (Molecular Probes, \#A-820, 1:6,000) was added to the primary antibody mix and 647 goat anti-mouse (Life Technologies \#Z25008, 1:200) secondary antibody was used instead of Cy3 goat anti-mouse. When Neurobiotin-350 (Vector Laboratories, \# SP-1155) was used for backfills, no modifications were required for the Brain immunostaining protocol. Brains were kept in the dark at all times.

Whole-mount preparation of adult antennae, maxillary palps and proboscis was performed as described in ref. 70 with minor modifications. On day 1, mosquitoes were immobilized on ice, their heads were cut off and placed in ZnFA fixative solution $\left(0.25 \% \mathrm{ZnCl}_{2}, 1 \%\right.$ formaldehyde, $135 \mathrm{mM} \mathrm{NaCl}, 1.2 \%$ sucrose and $0.03 \%$ Triton X-100) in $250 \mu \mathrm{l}$ PCR tubes for $20-24 \mathrm{~h}$ at RT in the dark. On day 2, the heads were washed twice for $15 \mathrm{~min}$ each with $\mathrm{HBS}$ buffer $(150 \mathrm{mM} \mathrm{NaCl}, 5 \mathrm{mM}$ $\mathrm{KCl}, 25 \mathrm{mM}$ sucrose, $10 \mathrm{mM}$ Hepes, $5 \mathrm{mM} \mathrm{CaCl}_{2}$ and $0.03 \%$ Triton X-100). Appendages were carefully cut off in HBS and placed in separate PCR tubes, based on gender and type of tissue (six PCR tubes in total for antennae, maxillary palps and proboscises of male and female mosquitoes). After a brief wash in HBS, the tissue was incubated in $80 \%$ methanol/20\% dimethyl sulfoxide (DMSO) solution for $1 \mathrm{~h}$ at RT, washed for $5 \mathrm{~min}$ in $0.1 \mathrm{M}$ Tris $\mathrm{pH} 7.4,0.03 \%$ Triton X-100 solution and incubated in blocking solution (PBS, 5\% NGS, $1 \%$ DMSO and $0.3 \%$ Triton $\mathrm{X}-100)$ for at least $3 \mathrm{~h}$ at RT. Next, the tissue was put in blocking solution containing the primary antibodies, the PCR tubes were placed in a buoyant perforated plastic pipette tip holder and allowed to float in a water bath sonicator (Bansonic 1,200, Branson, Danbury, CT) for 5 min (antennae and maxillary palps), or held with forceps and dipped into the water bath sonicator for 30-60 s (proboscises). Next, the PCR tubes were placed for 4 days at $4{ }^{\circ} \mathrm{C}$ in the dark. Sonification was repeated on day 3. On day 6, the tissue was washed at RT in PBS, $1 \%$ DMSO and $0.3 \%$ Triton X-100 for $2-3 \mathrm{~h}$. Secondary antibodies were added to blocking solution, the tubes were sonicated as described above and incubated for 3 days at $4{ }^{\circ} \mathrm{C}$ in the dark. On day 9 , the tissue was washed at RT in PBS, $1 \%$ DMSO and $0.3 \%$ Triton X-100 for 2-3 h, rinsed in PBS and mounted in Slow Fade Gold. To visualize CD8:GFP expression, we used a rat anti-CD8 primary antibody (Invitrogen \#MCD0800, 1:100). To visualize Orco protein, we used a rabbit antiDmOrco antibody ${ }^{29}$ (gift from Leslie Vosshall, Rockefeller University, 1:100). The secondary antibodies were Cy3 goat anti-rabbit (Jackson Immuno Research \#111-165-144, 1:200) and 633 goat anti-rat (Invitrogen \#A21094, 1:200). We did not use any green secondary antibody, to clearly visualize endogenous GFP fluorescence, driven by the transgenic reporter.

Whole-mount preparation of larval antennae and maxillary palps was performed similar to the adults. On day 1 , third and fourth instar larvae were separated by sex (females with red markings on their cuticle, males with pale cuticles; larvae with ambiguous markings were excluded). The larval heads were cut off and placed in ZnFA fixative solution in two separate $250 \mu \mathrm{l}$ PCR tubes (one for males and one for females) for $20-24 \mathrm{~h}$ at RT in the dark. The following steps were performed identically to the adult antennae and maxillary palps, with one exception: larval appendages remained attached to the heads throughout the staining protocol and were only dissected off immediately before mounting.

Cryosections of adult antennae were performed as described in ref. 17. On day 1 , heads with attached antennae of female mosquitoes were dissected and pre-fixed in $4 \%$ paraformaldehyde in. $03 \% \mathrm{PBT}$ at $4{ }^{\circ} \mathrm{C}$ for $30 \mathrm{~min}$, then rinsed three times with PBT and placed in a $25 \%$ sucrose solution in PBT overnight at $4^{\circ} \mathrm{C}$. On day 2 , samples were submerged in Tissue-Tek OCT compound (Sakura Finetek,

Torrance, CA) and frozen at $-80^{\circ} \mathrm{C}$ before being sectioned at $12 \mu \mathrm{m}$ on a Microm HM $500 \mathrm{M}$ cryostat (Microm International GmbH, Walldorf, Germany). Sections were collected on SuperFrost Plus slides (Fisher Scientific) and dried for $30 \mathrm{~min}$ at RT, then fixed in $4 \%$ paraformaldehyde in. $03 \%$ PBT at RT for $30 \mathrm{~min}$. Slides were rinsed three times with PBT at RT, blocked in PBT $+5 \%$ NGS for $1 \mathrm{~h}$ at RT and incubated with primary antibody in blocking solution overnight at $4{ }^{\circ} \mathrm{C}$ (rabbit anti-DmOrco ${ }^{29}$, gift from Leslie Vosshall, Rockefeller University, 1:100). On day 3, slides were rinsed three times at RT with PBT, then incubated with secondary antibody in block for $2 \mathrm{~h}$ at RT (Cy3 goat anti-rabbit, Jackson Immuno Research \#111-165-144, 1:200). No green secondary antibody was used, to visualize endogenous GFP fluorescence. Slides were rinsed three times with PBT at RT, then mounted in Slow Fade Gold.

Whole-animal imaging. Adult mosquitoes were anaesthetized at $4{ }^{\circ} \mathrm{C}$, placed on the ECHO therm chilling/heating plate (Torrey Pines Scientific) set to $0^{\circ} \mathrm{C}$ and imaged by a Zeiss SteREO Discovery.V8 microscope equipped with a GFP-470 and ds-Red filters and a Jenoptik ProgRes MF cool charge-coupled device camera. Images were acquired in ProgRes Mac Capture Pro 2.7 software and stored in *.tif format. Images that are compared with each other were obtained under identical hardware and software settings.

Petri dishes filled with water containing larval mosquitoes were placed on the ECHO therm chilling/heating plate set to $0^{\circ} \mathrm{C}$ and imaged as described for the adults.

Confocal imaging and analyses. Brains, head appendages and cryosections were imaged on a LSM 700 Zeiss confocal microscope at $512 \times 512$ pixel resolution, with $0.96 \mu \mathrm{m}$ or $2.37 \mu \mathrm{m} \mathrm{Z}$-steps. For illustration purposes, confocal images were processed in ImageJ to collapse Z-stacks into a single image; in some cases, the colour channels were swapped or duplicated.

No other image processing was performed on the confocal data.

To quantify Orco and mCD8-GFP co-expression in the adult (Supplementary Table 3), we used the 3D reconstruction software Amira (FEI, Oregon, USA) to manually outline cells stained with anti-Orco or anti-CD8 antibodies. Each outlined cell was assigned a separate label. Cells were marked independently in Orco (red) and in CD8 (blue) channels, creating two layers of labels in Amira. Next, 3D surfaces, corresponding to the outlined cells, were generated by the software and manually inspected as to the overlap of Orco and CD8 channels.

To quantify Orco (red) and mCD8-GFP (blue) co-staining with GFP fluorescence (green) in the larvae (Supplementary Tables 1 and 2), we used ImageJ with the Image5D plugin and Zen Zeiss software to mark and count cells in each of the three fluorescence channels.

To quantify the overlap of Orco staining (red) and endogenous GFP fluorescence (green) in the cryosectioned female antennae, we used ImageJ with the 
Image5D plugin and Zen Zeiss software to mark and count cells and sensilla in each channel.

The 3D reconstructions of the AL and SEZ were generated in Amira as described above. For the $\mathrm{AL}$, glomeruli were manually identified and labelled in the nc82 channel, and coloured based on whether they were also labelled in the Orco channel, the backfill channel or neither of these two. The correspondence between the data, presented in this study and in ref. 51 (Supplementary Table 5), was established by visual inspection of images in ref. 51 and finding a glomerulus in our data that appeared to have the closest anatomical location to each reported glomerulus. For the SEZ, glomerular-like structures were identified and labelled in the GFP channel, and volume rendering of the same channel was used to show processes. The 3D models are available (see below).

ALs showed high variability in appearance between individuals, in contrast to what we have observed in Drosophila ${ }^{75}$. Experimental conditions (for example, age, feeding and growth) were maintained as much as possible. Nonetheless, differences between AL samples probably arose due to a combination of technical (dissections and mounting) and biological reasons (for example, a rigid oesophagus pressing against ALs deforming glomeruli to different degrees). Future transgenic labelling of individual olfactory neuron classes will help address the source of glomerular variability.

Adult brain volumes were calculated as follows: we manually traced each confocal plane of nc82-stained male and female central brains in Amira (excluding optic lobes) and used the Amira software to generate 3D surfaces from the traces. These were used to calculate the enclosed surface volume of the central brain. AL volumes were calculated by summing $3 \mathrm{D}$ reconstructions of each glomerulus for each $\mathrm{AL}$

Data availability. The authors declare that data supporting the findings of this study are available with the article (and its Supplementary Information files). Source data and 3D models shown in Fig. 4a,b,d are available from the Potter Lab website at Johns Hopkins University School of Medicine (http://potterlab.johnshopkins.edu/natcomm2016). Additional data or information pertaining to this work are available from the corresponding author upon request.

\section{References}

1. WHO. World Malaria Report 2015. 1-280 (World Health Organization, 2015).

2. Alphey, L. Genetic control of mosquitoes. Annu. Rev. Entomol. 59, 205-224 (2014).

3. Foster, W. A. \& Takken, W. Nectar-related vs. human-related volatiles: behavioural response and choice by female and male Anopheles gambiae (Diptera: Culicidae) between emergence and first feeding. Bull. Entomol. Res. 94, 145-157 (2004)

4. Smallegange, R. C. \& van Gemert, G. J. Malaria infected mosquitoes express enhanced attraction to human odor. PLoS ONE 8, e63602 (2013).

5. Takken, W. The role of olfaction in host-seeking of mosquitoes: a review. Insect Sci. Appl. 12, 287-295 (1991).

6. Takken, W. \& Knols, B. G. Odor-mediated behavior of Afrotropical malaria mosquitoes. Annu. Rev. Entomol. 44, 131-157 (1999).

7. Zwiebel, L. J. \& Takken, W. Olfactory regulation of mosquito-host interactions. Insect Biochem. Mol. Biol. 34, 645-652 (2004).

8. Wilson, R. I. Early olfactory processing in Drosophila: mechanisms and principles. Annu. Rev. Neurosci. 36, 217-241 (2013).

9. Couto, A., Alenius, M. \& Dickson, B. J. Molecular, anatomical, and functional organization of the Drosophila olfactory system. Curr. Biol. 15, 1535-1547 (2005).

10. Fishilevich, E. \& Vosshall, L. B. Genetic and functional subdivision of the Drosophila antennal lobe. Curr. Biol. 15, 1548-1553 (2005).

11. Hallem, E. A., Fox, A. N., Zwiebel, L. J. \& Carlson, J. R. Olfaction: mosquito receptor for human-sweat odorant. Nature 427, 212-213 (2004).

12. Vosshall, L. B., Amrein, H., Morozov, P. S., Rzhetsky, A. \& Axel, R. A spatial map of olfactory receptor expression in the Drosophila antenna. Cell 96, 725-736 (1999)

13. Boo, K. S. Fine structure of the antennal sensory hairs in female Anopheles stephensi. Z. Parasitenkd. 61, 161-171 (1980).

14. McIver, S. \& Siemicki, R. Palpal sensilla of selected anopheline mosquitoes. J. Parasitol. 61, 535-538 (1975).

15. McIver, S. B. Sensilla mosquitoes (Diptera: Culicidae). J. Med. Entomol. 19, 489-535 (1982)

16. Fox, A. N., Pitts, R. J., Robertson, H. M., Carlson, J. R. \& Zwiebel, L. J. Candidate odorant receptors from the malaria vector mosquito Anopheles gambiae and evidence of down-regulation in response to blood feeding. Proc. Natl Acad. Sci. USA 98, 14693-14697 (2001).

17. Pitts, R. J., Fox, A. N. \& Zwiebel, L. J. A highly conserved candidate chemoreceptor expressed in both olfactory and gustatory tissues in the malaria vector Anopheles gambiae. Proc. Natl Acad. Sci. USA 101, 5058-5063 (2004).

18. Benton, R., Vannice, K. S., Gomez-Diaz, C. \& Vosshall, L. B. Variant ionotropic glutamate receptors as chemosensory receptors in Drosophila. Cell 136, 149-162 (2009).
19. Liu, C. et al. Distinct olfactory signaling mechanisms in the malaria vector mosquito Anopheles gambiae. PLoS Biol. 8, 1-17 (2010).

20. Hill, C. A. et al. G protein-coupled receptors in Anopheles gambiae. Science 298, 176-178 (2002)

21. Kwon, J. Y., Dahanukar, A., Weiss, L. A. \& Carlson, J. R. The molecular basis of CO2 reception in Drosophila. Proc. Natl Acad. Sci. USA 104, 3574-3578 (2007).

22. Lu, T. et al. Odor coding in the maxillary palp of the malaria vector mosquito Anopheles gambiae. Curr. Biol. 17, 1533-1544 (2007).

23. Scott, K. et al. A chemosensory gene family encoding candidate gustatory and olfactory receptors in Drosophila. Cell 104, 661-673 (2001).

24. Hallem, E. A., Ho, M. G. \& Carlson, J. R. The molecular basis of odor coding in the Drosophila antenna. Cell 117, 965-979 (2004).

25. Carey, A. F., Wang, G., Su, C.-Y., Zwiebel, L. J. \& Carlson, J. R. Odorant reception in the malaria mosquito Anopheles gambiae. Nature 464, 66-71 (2010).

26. Matthews, B. J., McBride, C. S., DeGennaro, M., Despo, O. \& Vosshall, L. B. The neurotranscriptome of the Aedes aegypti mosquito. BMC Genomics 17, 32 (2016).

27. Jones, W. D., Cayirlioglu, P., Grunwald Kadow, I. \& Vosshall, L. B. Two chemosensory receptors together mediate carbon dioxide detection in Drosophila. Nature 445, 86-90 (2006).

28. Benton, R., Sachse, S., Michnick, S. W. \& Vosshall, L. B. Atypical membrane topology and heteromeric function of Drosophila odorant receptors in vivo. PLoS Biol. 4, e20 (2006).

29. Larsson, M. C. et al. Or83b encodes a broadly expressed odorant receptor essential for Drosophila olfaction. Neuron 43, 703-714 (2004).

30. Xia, Y. et al. The molecular and cellular basis of olfactory-driven behavior in Anopheles gambiae larvae. Proc. Natl Acad. Sci. USA 105, 6433-6438 (2008).

31. Grant, A. J., Wigton, B. E., Aghajanian, J. G. \& O’Connell, R. J. Electrophysiological responses of receptor neurons in mosquito maxillary palp sensilla to carbon dioxide. J. Comp. Physiol. A 177, 389-396 (1995).

32. Brand, A. H. \& Perrimon, N. Targeted gene expression as a means of altering cell fates and generating dominant phenotypes. Development 118, 401-415 (1993).

33. Fischer, J. A., Giniger, E., Maniatis, T. \& Ptashne, M. GAL4 activates transcription in Drosophila. Nature 332, 853-856 (1988).

34. Potter, C. J., Tasic, B., Russler, E. V., Liang, L. \& Luo, L. The Q system: a repressible binary system for transgene expression, lineage tracing, and mosaic analysis. Cell 141, 536-548 (2010).

35. Riabinina, O. et al. Improved and expanded Q-system reagents for genetic manipulations. Nat. Methods 12, 219-222 (2015).

36. Venken, K. J. T., Simpson, J. H. \& Bellen, H. J. Genetic manipulation of genes and cells in the nervous system of the fruit fly. Neuron 72, 202-230 (2011).

37. Kokoza, V. A. \& Raikhel, A. S. Targeted gene expression in the transgenic Aedes aegypti using the binary Gal4-UAS system. Insect Biochem. Mol. Biol. 41, 637-644 (2011).

38. Lynd, A. \& Lycett, G. J. Development of the bi-partite Gal4-UAS system in the African malaria mosquito, Anopheles gambiae. PLoS ONE 7, e31552 (2012).

39. O’Brochta, D. A., Pilitt, K. L., Harrell, R. A., Aluvihare, C. \& Alford, R. T. Gal4-based enhancer-trapping in the malaria mosquito Anopheles stephensi. G3 (Bethesda) 2, 1305-1315 (2012).

40. Lee, T. \& Luo, L. Mosaic analysis with a repressible cell marker for studies of gene function in neuronal morphogenesis. Neuron 22, 451-461 (1999).

41. Pfeiffer, B. D. et al. Refinement of tools for targeted gene expression in Drosophila. Genetics 186, 735-755 (2010).

42. Levis, R., Hazelrigg, T. \& Rubin, G. M. Effects of genomic position on the expression of transduced copies of the white gene of Drosophila. Science 229, 558-561 (1985)

43. Weiler, K. S. \& Wakimoto, B. T. Heterochromatin and gene expression in Drosophila. Annu. Rev. Genet. 29, 577-605 (1995).

44. Schymura, D. et al. Antennal expression pattern of two olfactory receptors and an odorant binding protein implicated in host odor detection by the malaria vector Anopheles gambiae. Int. J. Biol. Sci. 6, 614-626 (2010).

45. Pitts, R. J., Rinker, D. C., Jones, P. L., Rokas, A. \& Zwiebel, L. J. Transcriptome profiling of chemosensory appendages in the malaria vector Anopheles gambiae reveals tissue- and sex-specific signatures of odor coding. BMC Genomics 12, $271(2011)$

46. Pitts, R. J. \& Zwiebel, L. J. Antennal sensilla of two female anopheline sibling species with differing host ranges. Malar. J. 5, 26 (2006).

47. Pitts, R. J., Liu, C., Zhou, X., Malpartida, J. C. \& Zwiebel, L. J. Odorant receptormediated sperm activation in disease vector mosquitoes. Proc. Natl Acad. Sci. USA 111, 2566-2571 (2014).

48. Vosshall, L. B., Wong, A. M. \& Axel, R. An olfactory sensory map in the fly brain. Cell 102, 147-159 (2000).

49. Anton, S. et al. Central projections of olfactory receptor neurons from single antennal and palpal sensilla in mosquitoes. Arthropod Struct. Dev. 32, 319-327 (2003). 
50. Ignell, R., Dekker, T., Ghaninia, M. \& Hansson, B. S. Neuronal architecture of the mosquito deutocerebrum. J. Comp. Neurol. 493, 207-240 (2005).

51. Ghaninia, M., Hansson, B. S. \& Ignell, R. The antennal lobe of the African malaria mosquito, Anopheles gambiae-innervation and three-dimensional reconstruction. Arthropod Struct. Dev. 36, 23-39 (2007).

52. Kwon, H.-W., Lu, T., Rutzler, M. \& Zwiebel, L. J. Olfactory responses in a gustatory organ of the malaria vector mosquito Anopheles gambiae. Proc. Natl Acad. Sci. USA 103, 13526-13531 (2006).

53. Hartl, M. et al. A new Prospero and microRNA-279 pathway restricts CO2 receptor neuron formation. J. Neurosci. 31, 15660-15673 (2011).

54. Jefferis, G. S. et al. Comprehensive maps of Drosophila higher olfactory centers: spatially segregated fruit and pheromone representation. Cell 128, 1187-1203 (2007).

55. Sparks, J. T., Bohbot, J. D. \& Dickens, J. C. The genetics of chemoreception in the labella and tarsi of Aedes aegypti. Insect Biochem. Mol. Biol. 48, 8-16 (2014).

56. Sparks, J. T., Vinyard, B. T. \& Dickens, J. C. Gustatory receptor expression in the labella and tarsi of Aedes aegypti. Insect Biochem. Mol. Biol. 43, 1161-1171 (2013).

57. Jung, J. W. et al. A novel olfactory pathway is essential for fast and efficient blood-feeding in mosquitoes. Sci. Rep. 5, 1-10 (2015).

58. Haverkamp, A. et al. Hawkmoths evaluate scenting flowers with the tip of their proboscis. Elife 5, e15039 (2016).

59. Diao, F. et al. Plug-and-play genetic access to Drosophila cell types using exchangeable exon cassettes. Cell Rep. 10, 1-43 (2015).

60. Chen, T. -W. et al. Ultrasensitive fluorescent proteins for imaging neuronal activity. Nature 499, 295-300 (2013).

61. de Bruyne, M., Clyne, P. J. \& Carlson, J. R. Odor coding in a model olfactory organ: the Drosophila maxillary palp. J. Neurosci. 19, 4520-4532 (1999).

62. Wang, J. W., Wong, A. M., Flores, J., Vosshall, L. B. \& Axel, R. Two-photon calcium imaging reveals an odor-evoked map of activity in the fly brain. Cell 112, 271-282 (2003).

63. Klapoetke, N. C. et al. Independent optical excitation of distinct neural populations. Nat. Methods 11, 338-346 (2014).

64. Lin, C.-C. \& Potter, C. J. Re-classification of Drosophila melanogaster trichoid and intermediate sensilla using fluorescence-guided single sensillum recording. PLOS ONE 10, e0139675 (2015).

65. Gallio, M., Ofstad, T. A., Macpherson, L. J., Wang, J. W. \& Zuker, C. S. The coding of temperature in the Drosophila brain. Cell 144, 614-624 (2011)

66. Corfas, R. A. \& Vosshall, L. B. The cation channel TRPA1 tunes mosquito thermotaxis to host temperatures. eLife 4, el1750 (2015).

67. Tauxe, G. M., MacWilliam, D., Boyle, S. M., Guda, T. \& Ray, A. Targeting a dual detector of skin and CO2 to modify mosquito host seeking. Cell 155, 1365-1379 (2013).

68. DeGennaro, M. et al. orco mutant mosquitoes lose strong preference for humans and are not repelled by volatile DEET. Nature 498, 487-491 (2013).

69. Karner, T., Kellner, I., Schultze, A., Breer, H. \& Krieger, Jr. Co-expression of six tightly clustered odorant receptor genes in the antenna of the malaria mosquito Anopheles gambiae. Front. Ecol. Evol. 3, 1-8 (2015).

70. Schultze, A., Pregitzer, P., Walter, M. F. \& Woods, D. F. The co-expression pattern of odorant binding proteins and olfactory receptors identify distinct trichoid sensilla on the antenna of the malaria mosquito. PLoS ONE 8, e69412 (2013).

71. Enjin, A. et al. Humidity sensing in Drosophila. Curr. Biol. 26, 1352-1358 (2016).
72. Li, X. et al. piggyBac internal sequences are necessary for efficient transformation of target genomes. Insect Mol. Biol. 14, 17-30 (2005).

73. Berdnik, D., Fan, A. P., Potter, C. J. \& Luo, L. MicroRNA processing pathway regulates olfactory neuron morphogenesis. Curr. Biol. 18, 1754-1759 (2008).

74. Siju, K. P., Hansson, B. S. \& Ignell, R. Immunocytochemical localization of serotonin in the central and peripheral chemosensory system of mosquitoes. Arthropod Struct. Dev. 37, 248-259 (2008).

75. Laissue, P. P. et al. Three-dimensional reconstruction of the antennal lobe in Drosophila melanogaster. J. Comp. Neurol. 405, 543-552 (1999).

76. Silbering, A. F. et al. Complementary function and integrated wiring of the evolutionarily distinct Drosophila olfactory subsystems. J. Neurosci. 31, 13357-13375 (2011).

\section{Acknowledgements}

This work was supported by a Johns Hopkins Medicine Discovery Fund and a Johns Hopkins Malaria Research Institute Pilot Grant. R.A. and D.O. were supported by a gran from the NIH NIAID (R01AI099060). We thank Leslie Vosshall for the anti-DmOrco antibody, Marcelo Jacobs-Lorena, Alex Kolodkin, Meg Younger and Conor McMeniman for discussions, and the Insect Transformation Facility (Rockville, MD) for Anopheles embryo injections. We thank Winter Okoth and Christopher Kizito at the Johns Hopkins School of Public Health for technical help with rearing mosquito lines.

\section{Author contributions}

O.R., D.T., C.-C.L. and E.M. performed the experiments and analysed data. R.A. and D.A.O. produced transgenic mosquitoes. O.R. and C.J.P. wrote the manuscript with input from all authors. C.J.P. supervised the project.

\section{Additional information}

Supplementary Information accompanies this paper at http://www.nature.com/ naturecommunications

Competing financial interests: The authors declare no competing financial interests.

Reprints and permission information is available online at http://npg.nature.com/ reprintsandpermissions/

How to cite this article: Riabinina, O. et al. Organization of olfactory centers in the malaria mosquito Anopheles gambiae. Nat. Commun. 7, 13010 doi: $10.1038 /$ ncomms13010 (2016).

This work is licensed under a Creative Commons Attribution 4.0 International License. The images or other third party material in this article are included in the article's Creative Commons license, unless indicated otherwise in the credit line; if the material is not included under the Creative Commons license, users will need to obtain permission from the license holder to reproduce the material. To view a copy of this license, visit http://creativecommons.org/licenses/by/4.0/

(C) The Author(s) 2016 